\title{
Fructus mume Ethanol Extract Prevents Inflammation and Normalizes the Septohippocampal Cholinergic System in a Rat Model of Chronic Cerebral Hypoperfusion
}

\author{
Min-Soo Kim, ${ }^{1,2, *}$ Ji Hye Bang, ${ }^{1,3, *}$ Jun Lee, Jung-Soo Han, ${ }^{2}$ Hyung Won Kang, ${ }^{4}$ and Won Kyung Jeon ${ }^{1}$ \\ ${ }^{1}$ Herbal Medicine Research Division, Korea Institute of Oriental Medicine, Daejeon, Korea. \\ ${ }^{2}$ Department of Biological Sciences, Konkuk University, Seoul, Korea. \\ ${ }^{3}$ Department of Internal Medicine, School of Medicine, Keimyung University, Daegu, Korea. \\ ${ }^{4}$ Department of Neuropsychiatry, College of Oriental Medicine, Wonkwang University, Jeonbuk, Republic of Korea.
}

\begin{abstract}
Fructus mume (F. mume), the unripe fruit of Prunus mume, has long been used in Asian countries to treat cough and chronic diarrhea. We previously reported that $F$. mume exerts anti-inflammatory effects in a model of chronic cerebral hypoperfusion $(\mathrm{CCH})$, a key etiological factor of vascular dementia $(\mathrm{VaD})$. The present study was performed to investigate the protective effects of an ethanolic extract of $F$. mume on the inflammatory response and cholinergic dysfunction in a model of $\mathrm{CCH}$ induced by bilateral common carotid artery occlusion (BCCAo) in Wistar rats. Rats were assigned to three treatment groups: sham plus vehicle, BCCAo plus vehicle, and BCCAo plus $F$. mume extract $(200 \mathrm{mg} / \mathrm{kg})$. F. mume was administered by oral gavage from days 21 to 42 following BCCAo. Glial cell numbers were measured in the white matter and hippocampus. The hippocampal expressions of proinflammatory cytokines, angiotensin-II (Ang-II), receptor for advanced glycation end products (RAGE), and mitogen-activated protein kinase (MAPKs) were also evaluated. Choline acetyltransferase (ChAT) levels in the hippocampus and basal forebrain were examined. Rats with BCCAo showed an increase in the number of glial cells and levels of proinflammatory cytokines, Ang-II, RAGE, and MAPKs, all of which were significantly attenuated by $F$. mume treatment. F. mume administration also restored ChAT expression in the basal forebrain and hippocampus following chronic BCCAo. These results suggest that $F$. mume is a potentially valuable drug or nutraceutical for the treatment of $\mathrm{VaD}$.
\end{abstract}

KEY WORDS: • bilateral common carotid artery occlusion • choline acetyltransferase $\bullet$ chronic cerebral hypoperfusion - Fructus mume • neuroinflammation

\section{INTRODUCTION}

V ASCUlAR DEMENTIA (VAD) accounts for $\sim 15-25 \%$ of dementia cases and occurs when the blood supply to the brain is reduced by vessel occlusion. ${ }^{1,2}$ Although the estimated expenses of $\mathrm{VaD}$ care and treatment are quite large, the underlying mechanisms and pathogenesis remain unclear. Previous research has shown that a long-lasting decrease in cerebral blood flow caused by chronic cerebral hypoperfusion $(\mathrm{CCH})$ is a key etiological factor in $\mathrm{VaD} .^{3,4}$ Many animal models have been developed to study the

\footnotetext{
*These authors contributed equally to this article.
}

Manuscript received 8 May 2015. Revision accepted 17 November 2015

Address correspondence to: Won Kyung Jeon, PhD, Herbal Medicine Research Division, Korea Institute of Oriental Medicine, 1672 Yuseongdae-ro, Yuseong-gu, Daejeon 34054, Republic of Korea, E-mail: wkjeon@kiom.re.kr pathogenesis and therapeutics of $\mathrm{VaD}$. Among these, the permanent bilateral common carotid artery occlusion (BCCAo) animal model has been widely used. In this model, blood flow to the cerebral cortex is reduced to $33-45 \%$ of normal levels 2 days after BCCAo. ${ }^{5}$ Inflammatory responses are a highly common manifestation of $\mathrm{VaD}$. We previously reported that neuroinflammatory responses are induced following chronic BCCAo in the white matter and hippocampus, which are particularly vulnerable to $\mathrm{CCH}^{6,7}$ Moreover, blood samples from patients with $\mathrm{VaD}$ contained higher levels of inflammatory molecules than did control samples. ${ }^{8,9}$

Another important feature of $\mathrm{VaD}$ is cholinergic dysfunction. Acetylcholine (ACh) is a neurotransmitter in the central nervous system important for synaptic transmission, synaptic plasticity, and memory formation. ${ }^{10}$ Cholinergic innervation of the basal forebrain provides projections to the cortex and hippocampus, ${ }^{11}$ brain regions involved in

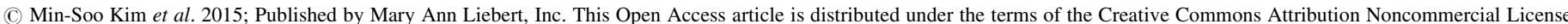

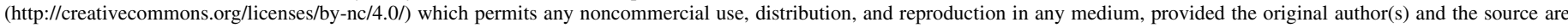
credited. 
learning and memory. It is therefore believed that cholinergic dysfunction induced by $\mathrm{VaD}$ can lead to cognitive impairment. ${ }^{12}$ Several studies have reported a decrease in the level of choline acetyltransferase (ChAT), a biosynthetic enzyme for ACh, in the basal forebrain and hippocampus of rats with chronic BCCAo. ${ }^{13,14}$ Taken together, these studies suggest that protecting against inflammation and cholinergic dysfunction following $\mathrm{CCH}$ might be an effective intervention for $\mathrm{VaD}$.

Fructus mume (F. mume), an unripe fruit of Prunus mume (P. mume), is often used in Asian countries to treat ulceration, chronic diarrhea, and gastrointestinal diseases. ${ }^{15}$ Previous studies have demonstrated the in vivo and in vitro therapeutic efficacy of $F$. mume in several pathological conditions. For example, $F$. mume blocked expression of proinflammatory molecules in lipopolysaccharide-stimulated RAW 264.7 cells (a mouse leukemic monocyte-macrophage cell line). ${ }^{16}$ Administration of a $P$. mume mixture in colitis-induced mice reduced the expression of inflammatory cytokines such as cyclooxygenase-2 (cox-2) and interleukin-4 (IL-4). ${ }^{17}$ In addition, $F$. mume treatment reversed scopolamine-induced cognitive disability by regulating cholinergic neurons. ${ }^{18}$ These studies indicate that $F$. mume could regulate both inflammatory responses and the cholinergic system.

We previously reported that a $F$. mume water extract attenuated activation of microglia and phosphorylated extracellular signal-regulated kinase (p-ERK) in the hippocampus of rats with chronic BCCAo. ${ }^{19}$ In general, naturally derived drugs are extracted with not only water but also with ethanol, which results in the extraction of both polar and nonpolar compounds. ${ }^{20}$ Since $F$. mume shows anti-inflammatory effects, ethanol extracts of $F$. mume (E. F. mume) are also expected to treat the pathogenic processes associated with $\mathrm{VaD}$. The present study was therefore designed to examine the protective role of E. F. mume on inflammation and cholinergic dysfunction induced by $\mathrm{CCH}$. The anti-inflammatory effects of E. F. mume on glial cell activation, proinflammatory cytokines, the receptor for advanced glycation end products (RAGE), angiotensin-II (Ang-II), and mitogenactivated protein kinase (MAPK) were investigated in rats with chronic BCCAo. In addition, we evaluated ChAT expression as a cholinergic marker in the medial septum and hippocampus.

\section{MATERIALS AND METHODS}

\section{Animals}

Twenty-six male Wistar rats were used in the chronic BCCAo experiment (12 weeks old; Charles River Co., Gapyeong, Korea). For 2 weeks at the beginning of the experiment, rats were housed in a vivarium at the Korea Institute of Oriental Medicine (KIOM) under controlled temperature $\left(22^{\circ} \mathrm{C} \pm 1{ }^{\circ} \mathrm{C}\right)$ and humidity $(55 \% \pm 10 \%)$ with a 12-h light/12-h dark cycle. Food and water were given ad libitum to all rats. All experimental procedures described in this report were approved by the Institutional Animal Care and Use Committee of the KIOM (2013-019).

\section{BCCAo surgery}

Rats were anesthetized with $5 \%$ isoflurane in a mixture of $30 \%$ oxygen and $70 \%$ nitrogen. BCCAo surgery was performed as described previously. ${ }^{7}$ A midline incision was performed to expose both common carotid arteries, which were then tightly double-ligated using silk sutures (size 4-0). Control rats were subjected to a sham operation without occlusion. During the surgical procedure, rat body temperatures were maintained at $37.0^{\circ} \mathrm{C} \pm 0.5^{\circ} \mathrm{C}$.

\section{Preparation of $\mathrm{F}$. mume extracts and administration}

F. mume was produced in Korea and obtained from a commercial supplier (Omniherb, Daegu, Korea). The species was identified by the Herbal Quality Control Team and deposited at the Creative Research Laboratory of the KIOM. F. mume was dried and extracted in 70\% ethanol using an ultrasound-assisted extractor (OM30-EP; Sonimedi, Wonju, Korea). The extract was concentrated by vacuum using a rotary evaporator after filtration. A high-performance liquid chromatography assay was carried out using a Waters 2695 Alliance HPLC System ${ }^{21}$ (Waters, Milford, MA). The mobile phase comprised acetonitrile (Buffer A) and water (Buffer B) with the following stepwise gradient elution protocol: $0 \mathrm{~min}, 100 \% \mathrm{~B} ; 15 \mathrm{~min}$, $90 \% \mathrm{~B} ; 40 \mathrm{~min}, 0 \% \mathrm{~B}$. The flow rate was $1.0 \mathrm{~mL} / \mathrm{min}$. E. F. mume contained prunasin, $\alpha$-hydroxy-benzeneacetamide, benzyl- $O-\beta$-Dglucopyranoside, benzyl- $O$ - $\alpha$-L-arabinopyranosyl- $\beta$-Dglucopyranoside, 5-hydroxymethyl-2-furaldehyde, and benzyl- $O$ - $\beta$-D-xylopyranosyl- $\beta$-D-glucopyranoside.

In the present study, the rats were divided into three groups: a sham-operated group administered saline $(n=8)$; a BCCAo group administered saline $(n=10)$; and a BCCAo group administered the $F$. mume extract $(200 \mathrm{mg} / \mathrm{kg}, n=8)$. $F$. mume was dissolved in saline and administered by oral gavage (p.o.). Treatments with saline or $F$. mume were administered from days 21 to 42 following BCCAo or sham surgery. During oral administration, rats displayed no toxic effects as identified by changes in general behavior and mortality.

\section{Western blot analysis}

Protein samples of hippocampal tissue were prepared and homogenized in cold lysis buffer containing $25 \mathrm{mM}$ Tris $\mathrm{HCl}$ $\mathrm{pH} 7.6,150 \mathrm{mM} \mathrm{NaCl}, 1 \%$ nonyl phenoxypolyethoxylethanol, $1 \%$ sodium deoxycholate, $0.1 \%$ sodium dodecyl sulfate, (Thermo Scientific, Waltham, MA), and protease and phosphatase inhibitor cocktail solutions (GenDEPOT, Barker, TX). Homogenates were centrifuged at 20,000 $g$ for $30 \mathrm{~min}$ at $4^{\circ} \mathrm{C}$, and the supernatants were harvested and stored at $-70^{\circ} \mathrm{C}$. Protein concentrations were determined using the BCA assay (Thermo Scientific). Protein samples $(40 \mu \mathrm{g})$ were then separated by $8-12 \%$ sodium dodecyl sulfate-polyacrylamide gel electrophoresis and transferred to a polyvinylidene difluoride membrane, which was subsequently incubated in primary antibodies against interleukin-1 $\beta$ (IL-1 $\beta$; Millipore Corporation, Billerica, MA), cox-2 (Abcam, San Francisco, CA), RAGE (Abcam), Ang-II (Abcam), interleukin-6 (IL-6; Abcam), ERK 
(Cell Signaling, Danvers, MA), p38 MAPK (Cell Signaling), c-Jun N-terminal kinases (JNK; Cell Signaling), or ChAT (Millipore Corporation). Glyceraldehyde-3-phosphate dehydrogenase (GAPDH; Santa Cruz Biotechnologies, Santa Cruz, CA) was used as an internal control. A goat anti-rabbit horseradish peroxidase-conjugated secondary antibody (Cell Signaling) was then added. Detection was carried out using an enhanced chemiluminescence system (Thermo Scientific) with a Lumino Image Analyzer (Las-4000; Fujifilm, Tokyo, Japan). Densitometry was performed for specific markers normalized to GAPDH using Multigage software (Fujifilm).

\section{Immunohistochemistry}

Whole brains were postfixed in $4 \%$ paraformaldehyde for 7 days, cryoprotected in phosphate-buffered saline (PBS) containing $30 \%$ sucrose for 21 days at $4{ }^{\circ} \mathrm{C}$, and then stored at $-70^{\circ} \mathrm{C}$. Brain cryosections $(40 \mu \mathrm{m})$ were incubated with ionized calcium binding adaptor molecule-1 (Iba-1; Wako, Tokyo, Japan), glial fibrillary acidic protein (GFAP; Abcam), and NeuN (Millipore Corporation) in 3\% casein in PBS containing $0.1 \%$ Triton-X 100 overnight at $4^{\circ} \mathrm{C}$. Cryosections were then incubated with an anti-rabbit $\operatorname{IgG}$ secondary antibody (Cell Signaling). Last, stained sections were treated with a Vector DAB kit (Vector Laboratories,
Burlingame, CA) for peroxidase-mediated staining and mounted onto resin-coated slides using the Permount reagent (Fisher Scientific, Pittsburgh, PA). The stained sections were examined under light microscopy $(\mathrm{Bx} 51$; Olympus, Tokyo, Japan), and Iba- and GFAP-positive glial cell numbers were assessed in one region of interest (ROI) of $0.03 \mathrm{~mm}^{2}$ per one section in the corpus callosum, fimbria, optic tract, and hippocampus (including the CA1, CA3, and dentate gyrus [DG]). The number of ChAT-positive cholinergic neurons was assessed in an ROI of $0.03 \mathrm{~mm}^{2}$ per one section in the basal forebrain. Three sections were selected per rat, and cell counts are presented as mean numbers of Iba-1-, GFAP-, and ChAT-positive cells in each region, respectively.

\section{Statistical analysis}

Differences between groups were considered significant at $P<.05$. A one-way analysis of variance (ANOVA) and post hoc test (least significant difference) were performed to determine the effects of $F$. mume administration on alterations in the numbers of Iba-1-, GFAP-, and ChAT-positive cells, as well as on changes in protein expression levels induced by chronic BCCAo. All data are presented as mean \pm standard deviation (SD).
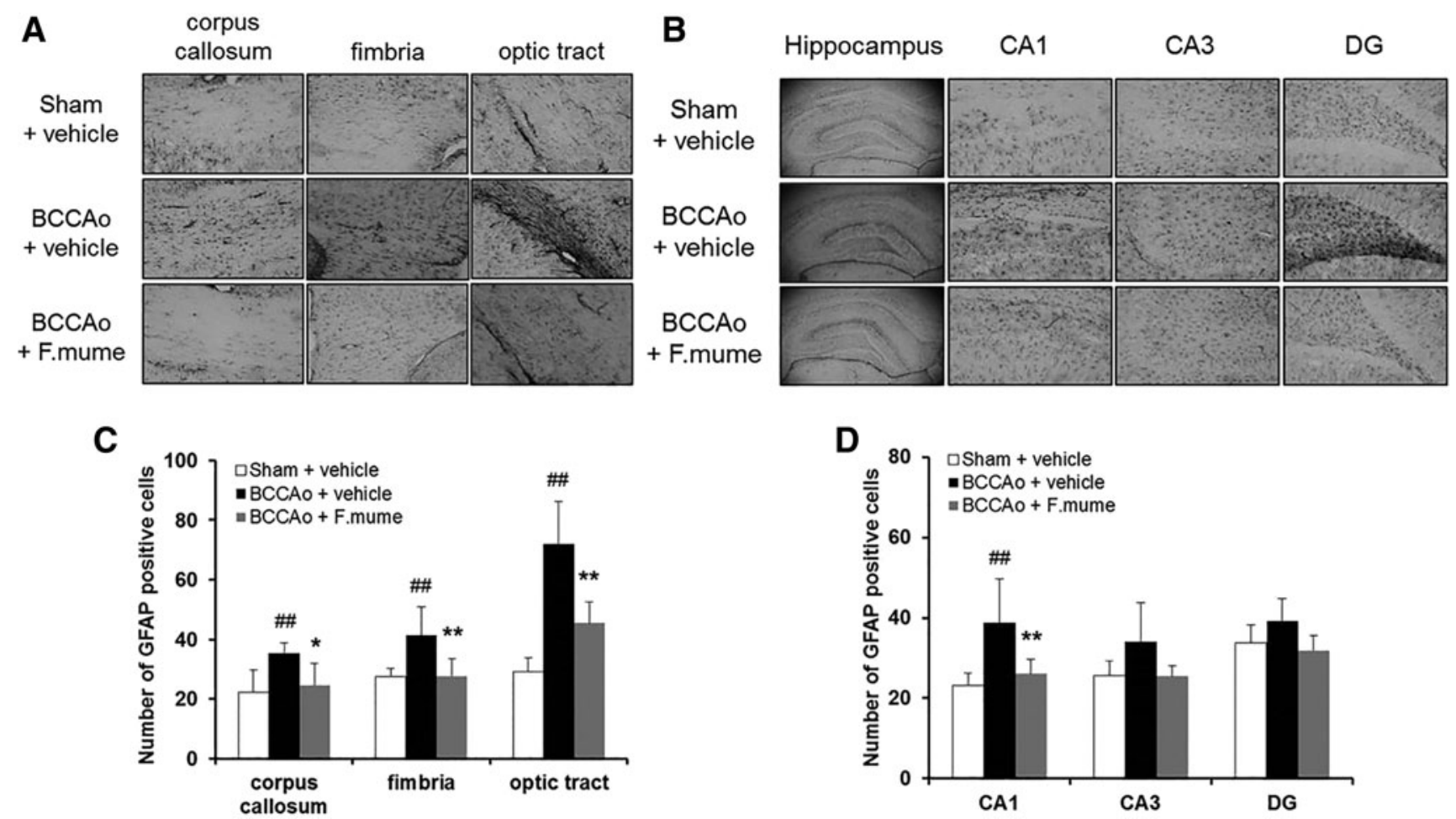

FIG. 1. Effect of Fructus mume on chronic BCCAo-induced astrocyte activation in the white matter and subregions of the hippocampus. (A, B) Photomicrographs of the immunohistochemical staining for GFAP. GFAP staining in the corpus callosum, fimbria, and optic tract (A). GFAP staining in the hippocampal CA1, CA3, and DG subregions (B). (C, D) Histograms depicting the absolute numbers of GFAP-immunopositive astrocytes in the sham-operated $(n=6)$, BCCAo plus vehicle $(n=8)$, and BCCAo plus $F$. mume $(n=8)$ groups. Values are expressed as the mean \pm SD. ${ }^{\# \#} P<.01$ compared with sham plus vehicle; $* P<.05, * * P<.01$ compared with BCCAo plus vehicle. Original magnification was $40 \times$ for the whole hippocampus and $200 \times$ for other regions. BCCAo, bilateral common carotid artery occlusion; DG, dentate gyrus; GFAP, glial fibrillary acidic protein. 


\section{RESULTS}

F. mume extracts inhibited white matter and hippocampal glial cell activation induced by chronic BCCAo

Expression of glial cells is increased following $\mathrm{CCH}^{22}$ To examine the effects of E. F. mume on glial activation, the numbers of GFAP- and Iba-1-positive cells were measured in the corpus callosum, fimbria, optic tract, and hippocampus (including the CA1, CA3, and DG subregions). Figure 1 shows the expression of GFAP in all the three treatment groups. A one-way ANOVA revealed significant between-group effects in all regions investigated $\left(\mathrm{F}_{(2,19)} \geq 4.644, P<.023\right)$. According to post hoc analyses, GFAP-positive astrocytes were significantly increased in the corpus callosum, fimbria, optic tract, and CA1 (but not CA3 or DG) of the hippocampus in the BCCAo group compared with the sham group $(P<.05)$. Administration of E. F. mume $(200 \mathrm{mg} / \mathrm{kg})$ significantly decreased GFAP levels relative to the BCCAo group $(P<.05$, Fig. 1).

The number of Iba-1-positive microglia was also estimated, as shown in Figure 2. A one-way ANOVA revealed significant group effects in the corpus callosum, fimbria, optic tract, and hippocampal CA1 regions $\left(\mathrm{F}_{(2,23)} \geq 4.488\right.$, $P<.023)$. Subsequent post hoc analyses revealed that $\mathrm{E}$. $F$. mume significantly inhibited the increase of Iba-1positive microglia in the white matter and CA1 of the hippocampus in BCCAo rats $(P<.05$, Fig. 2). These results were consistent with previous findings using $F$. mume water extracts. ${ }^{19}$ In addition, we observed neuronal cell death in subregions of the hippocampus by counting NeuN-positive cells to assess neurotoxicity. There were no clear signs of neuronal cell death after chronic BCCAo, and $F$. mume did not affect neuronal cell death (data not shown).

\section{F. mume attenuated the hippocampal expression of} proinflammatory cytokines induced by chronic BCCAo

Our previous report showed that the increase in levels of neuroinflammatory biomolecules, which contribute to neuronal dystrophy, ${ }^{23}$ is noticeable after chronic BCCAO in rats. ${ }^{6}$ We therefore measured the protein levels of major proinflammatory cytokines in the hippocampus by Western blot, including cox-2, IL-1 $\beta$, and IL-6. According to oneway ANOVA, significant between-group effects in cox-2, IL-1 $\beta$, and IL-6 levels were shown $\left(\mathrm{F}_{(2,19)} \geq 10.892, P<\right.$ .001). In the BCCAo group, the levels of these cytokines were higher when compared with the sham group $(P<.05)$; however, BCCAo-treated rats receiving E. F. mume had significantly lower proinflammatory cytokines than rats treated with BCCAo alone $(P<.05$, Fig. 3$)$.

F. mume prevented increases in hippocampal expression of Ang-II and RAGE induced by chronic BCCAo

Ang-II and RAGE promote the enhancement of proinflammatory cytokines. ${ }^{24,25}$ The effects of E. F. mume on
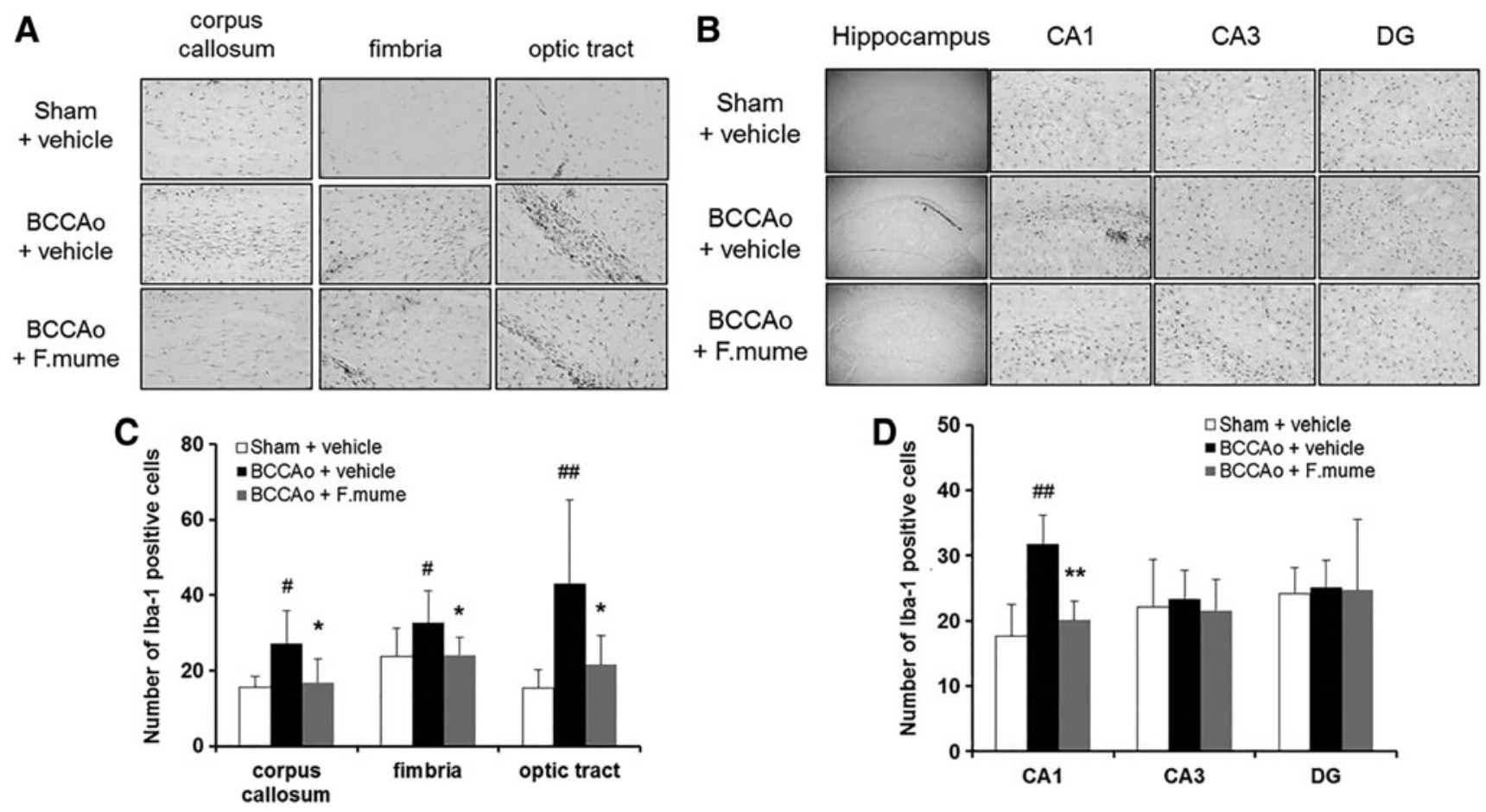

FIG. 2. Effect of F. mume on the chronic BCCAo-induced microglial activation in the white matter and subregions of the hippocampus. (A, B) Photomicrographs of the immunohistochemical staining for Iba-1. Iba-1 staining in the corpus callosum, fimbria, and optic tract (A). Iba-1 staining in the hippocampal CA1, CA3, and DG subregions (B). (C, D) Histograms depicting the absolute numbers of Iba-1-immunopositive microglia in the sham-operated $(n=8)$, BCCAo plus vehicle $(n=10)$, and BCCAo plus $F$. mume $(n=8)$ groups. Values are expressed as the mean \pm SD. ${ }^{\#} P<.05,{ }^{\# \#} P<.01$ compared with sham plus vehicle; $* P<.05, * * P<.01$ compared with BCCAo plus vehicle. Original magnification was $40 \times$ for the whole hippocampus and $200 \times$ for other regions. 
A

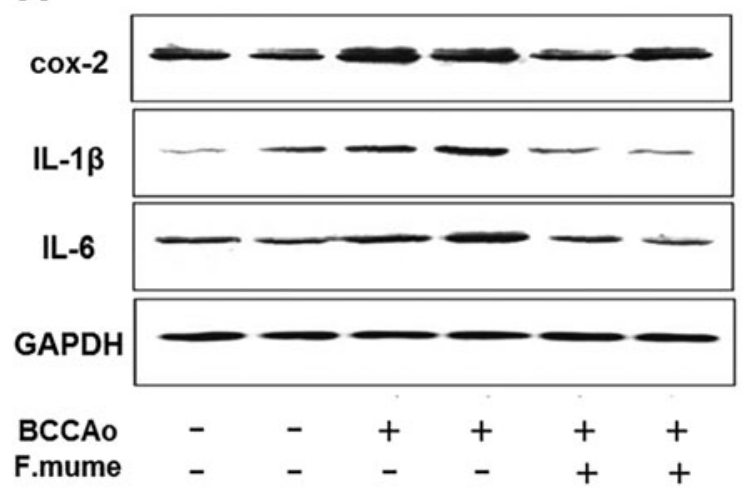

B

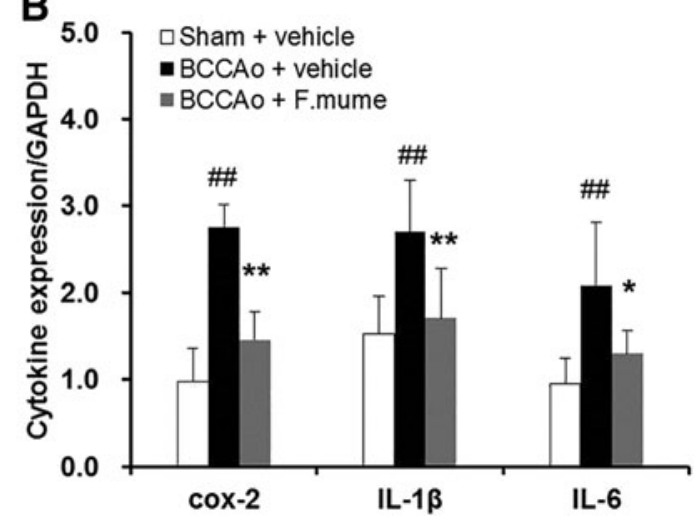

FIG. 3. Effect of F. mume on hippocampal expression levels of proinflammatory cytokines in chronic BCCAo-treated rats. (A) Representative Western blot of cox-2, IL-1 $\beta$, and IL-6. (B) Expression levels of cox-2, IL-1 $\beta$, and IL-6 in the sham-operated $(n=8)$, BCCAo plus vehicle $(n=8)$, and BCCAo plus $F$. mume $(n=6)$ groups. Expression levels were normalized to GAPDH. Values are expressed as mean \pm SD. ${ }^{\# \#} P<.01$ compared with sham plus vehicle; $* P<.05, * * P<.01$ compared with BCCAo plus vehicle. Cox-2, cyclooxygenase-2; GAPDH, glyceraldehyde-3-phosphate dehydrogenase; IL-1 $\beta$, interleukin- $\beta$; IL-6, interleukin-6.

Ang-II and RAGE protein expression were therefore determined in the hippocampus. Among the three rat treatment categories, a one-way ANOVA showed significant betweengroup effects for Ang-II and RAGE $\left(\mathrm{F}_{(2,19)} \geq 10.939, P<.001\right)$. Subsequent post hoc analyses revealed that chronic BCCAo increased the expression of Ang-II and RAGE in the hippocampus compared with sham $(P<.05)$. However, BCCAotreated rats receiving E. F. mume showed a significant decrease in Ang-II and RAGE levels $(P<.05$, Fig. 4).

F. mume downregulated hippocampal ERK and p38 MAPK signaling induced by chronic BCCAo

Since MAPKs are associated with proinflammatory cytokine expression, ${ }^{26,27}$ the levels of phosphorylated (p-ERK, p-p38, and p-JNK) and unphosphorylated (ERK, p38, and JNK) MAPKs were measured in the hippocampus to examine the effects of E. F. mume administration. As shown in Figure 6, significant between-group effects were detected for $\mathrm{p}$-ERK and $\mathrm{p}-\mathrm{p} 38\left(\mathrm{~F}_{(2,19)} \geq 6.351, P<.008\right)$. No significant differences were found for $\mathrm{p}$-JNK. After chronic BCCAo surgery, protein levels of p-ERK and p-p38 were significantly increased; however, these increases were not observed in the BCCAo-treated rats administered E. $F$. mume $(P<.05$, Fig. 5).

F. mume extracts increased expression of ChAT in the medial septum and hippocampus of rats treated with chronic BCCAo

Alteration of MAPK signaling is related to dysfunction of the cholinergic system. ${ }^{28}$ To investigate the effect of $\mathrm{E}$. $F$. mume on cholinergic deficiency induced by $\mathrm{CCH}$, expression of ChAT was measured in the basal forebrain using immunohistochemistry (Fig. 6A, B). A one-way ANOVA revealed significant between-group effects $\left(\mathrm{F}_{(2,9)}=23.187\right.$, $P<.001)$. Subsequent post hoc analyses indicated that the number of ChAT-positive cells was increased in the
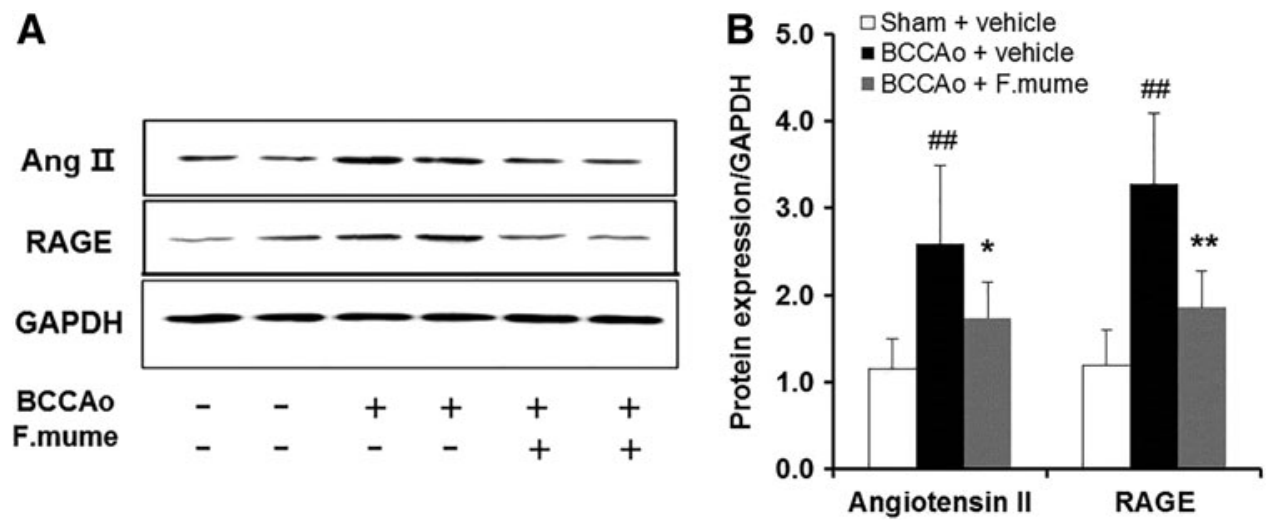

FIG. 4. Effect of F. mume on hippocampal expression levels of Ang-II and RAGE in rats with chronic BCCAo. (A) Representative Western blots depicting protein levels of Ang-II and RAGE. (B) The expression levels of Ang-II and RAGE in the sham-operated group ( $n=8$ ), BCCAo plus vehicle group $(n=8)$, and BCCAo plus $F$. mume group $(n=6)$. Expression levels were normalized to GAPDH. Values are expressed as mean \pm SD. ${ }^{\#} P<.01$ compared with sham plus vehicle group; $* P<.05$, $* * P<.01$ compared with BCCAo plus vehicle group. Ang-II, angiotensinII; RAGE, receptor for advanced glycation end products. 
A

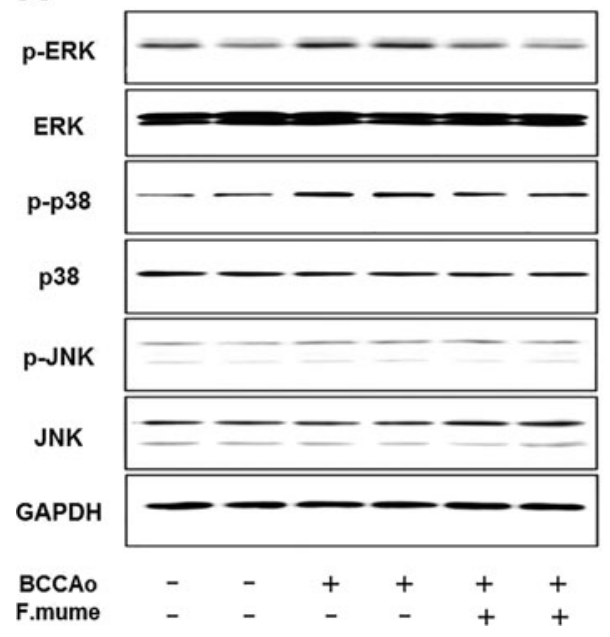

B

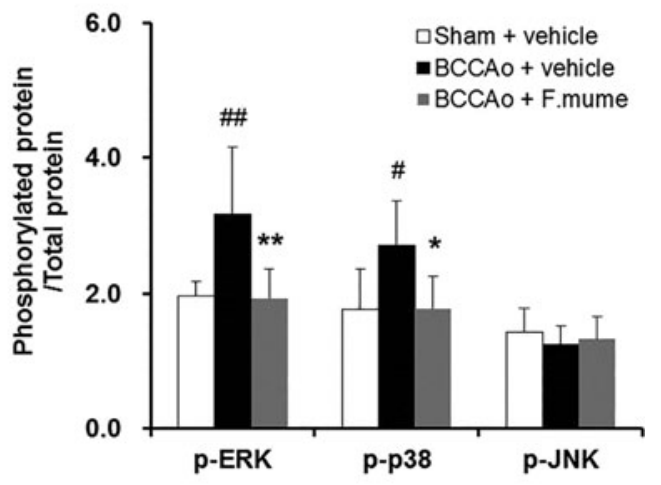

FIG. 5. Effect of F. mume on the hippocampal expression of MAPKs in rats with chronic BCCAo. (A) Representative Western blot of MAPKs (ERK, p38, and JNK) and phosphorylated MAPKs (phospho-ERK, phospho-p38, and phospho-JNK). (B) The expression levels of MAPKs and phosphorylated MAPKs in the sham-operated $(n=8)$, BCCAo plus vehicle $(n=8)$, and BCCAo plus $F$. mume groups $(n=6)$. Values are expressed as mean $\pm \mathrm{SD}$. ${ }^{\#} P<.05$, ${ }^{\#} P<.01$ compared with sham plus vehicle; $* P<.05, * * P<.01$ compared with BCCAo plus vehicle. ERK, extracellular signal-regulated kinase; JNK, c-Jun Nterminal kinase; MAPK, mitogenactivated protein kinase.
BCCAo treated with E. F. mume compared with the vehicle-treated BCCAo group $(P<.05)$. We also estimated hippocampal ChAT expression by Western blot (Fig. 6C, D). ANOVA revealed significant between-group effects $\left(\mathrm{F}_{(2,13)}=27.826, P<.001\right)$. Similar to results in the medial septum, E. F. mume administration upregulated the decrease of hippocampal ChAT induced by chronic BCCAo $(P<.05)$.

\section{DISCUSSION}

We previously reported that $F$. mume water extracts improved spatial memory and downregulated microglia and p-ERK activation post-BCCAo, ${ }^{19}$ suggesting that $F$. mume prevents the inflammatory response induced by VaD. Naturally derived drugs are often extracted using both ethanol and water. ${ }^{29,30}$ Using both water and ethanol extracts yields potentially therapeutic polar and nonpolar compounds. We recently have found that $F$. mume ethanol extracts attenuate scopolamine-induced memory impairment in mice. ${ }^{18}$ We therefore further investigated the effects of $F$. mume ethanol extracts on $\mathrm{CCH}$ in the present study. Given that $F$. mume water extracts exert antiinflammatory effects, ${ }^{19}$ it is also reasonable to hypothesize that E. F. mume ameliorates the inflammation and pathology associated with $\mathrm{VaD}$.
A

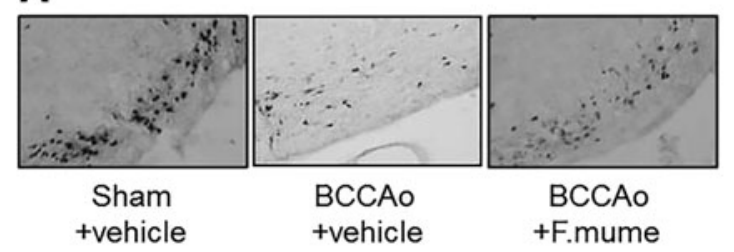

C

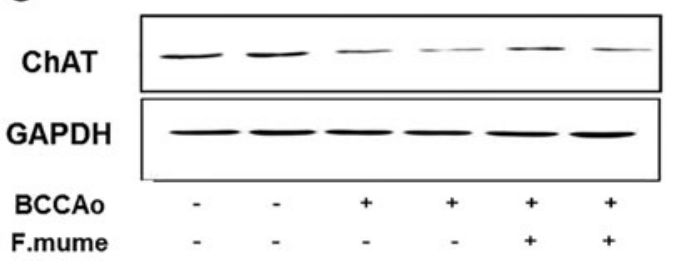

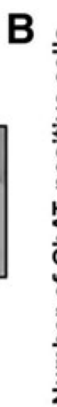
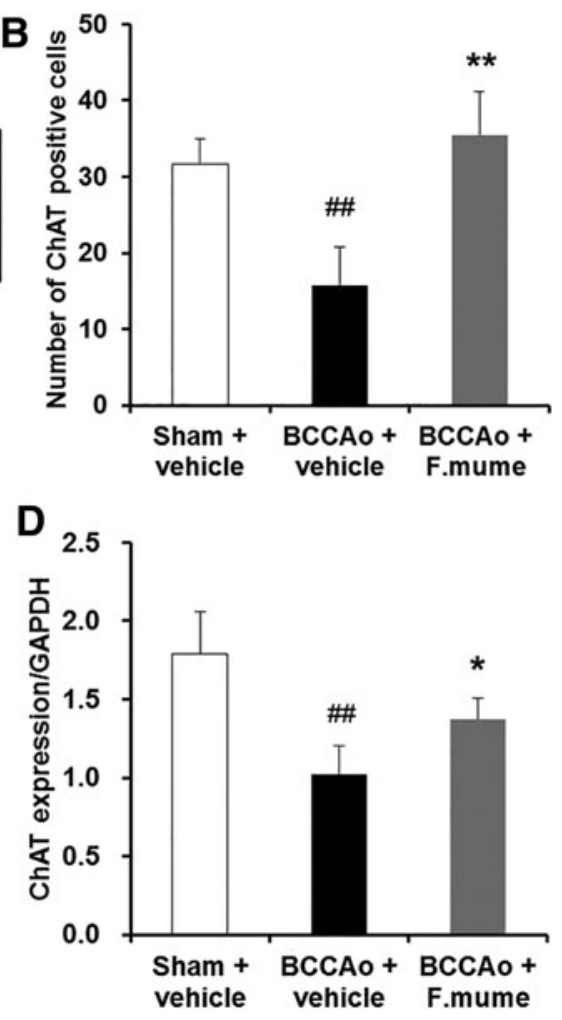

FIG. 6. Effect of $F$. mume on the septohippocampal cholinergic system in rats with chronic BCCAo. (A) Photomicrographs of immunohistochemical staining for ChAT in the basal forebrain. (B) Histograms depicting the absolute numbers of ChAT-positive cells in the sham-operated $(n=4)$, BCCAo plus vehicle $(n=4)$, and BCCAo plus $F$. mume groups $(n=4)$. (C) Representative Western blot of ChAT in the hippocampus. (D) The expression levels of hippocampal ChAT in the sham-operated $(n=8)$, BCCAo plus vehicle $(n=8)$, and BCCAo plus $F$. mume groups $(n=6)$. Expression levels were normalized to GAPDH. Values are expressed as mean $\pm \mathrm{SD}$. ${ }^{\# \#} P<.01$ compared with sham plus vehicle; $* P<.05, * * P<.01$ compared with BCCAo plus vehicle. Original magnification was $200 \times$. ChAT, choline acetyltransferase. 
Chemical analysis of $F$. mume extracts by HPLC revealed several bioactive compounds, such as prunasin, benzyl- $O-\beta$-Dglucopyranoside, and 5-hydroxymethyl-2-furaldehyde. ${ }^{21}$ Some studies have revealed that these compounds could have beneficial effects. Prunasin and benzyl- $O$ - $\beta$-D-glucopyranoside isolated from Prunus persica seeds have an antitumor promoting activity, which inhibit Epstein-Barr virus early antigen activation. ${ }^{31}$ Another $F$. mume constituent, 5-hydroxymethyl2-furaldehyde, is also a major component of Jaeumganghwatang, which is a herbal prescription and has anti-inflammatory activity. ${ }^{32}$ In addition, ursolic acid present in $F$. mume exerts antitumor actions, as shown by its inhibition of HIMeg and HL-60 cell proliferations. ${ }^{33}$

It has been well documented that inflammatory responses, including activation of glial cells, proinflammatory cytokines, and MAPKs, are caused by chronic BCCAo. ${ }^{7,34}$ The major proinflammatory cytokines IL- $1 \beta$ and IL-6 may also contribute to neuroinflammation. ${ }^{35}$ Moreover, the MAPK signaling pathway is crucial for the release of proinflammatory mediators. ${ }^{36}$ We reported previously that $F$. mume water extracts significantly attenuate microglial activation induced by chronic BCCAo in the hippocampal CA1 region and fimbria. ${ }^{19}$ In line with previous reports, we found that administration of E. F. mume decreased Iba-1-positive microglia in the hippocampal CA1, fimbria, corpus callosum, and optic tract. We also found that E. F. mume treatment decreased GFAP-positive astrocytes in the white matter and hippocampal CA1 region, and reduced hippocampal proinflammatory cytokine levels. In addition, BCCAo rats administered E. F. mume had lower hippocampal levels of p-p38 and p-ERK than those treated with BCCAo alone. Thus, these results indicate that E. F. mume also exerts anti-inflammatory effects on $\mathrm{CCH}$.

We further investigated whether E. F. mume affects AngII and RAGE expression levels in the hippocampus. Ang-II is a peptide hormone, which can cause platelet aggregation. ${ }^{37}$ It has been revealed that proinflammatory cytokines, including IL- $1 \beta$, were inhibited in hypertensive rats treated with Ang-II receptor type 1 antagonist. ${ }^{24}$ RAGE is implicated in Alzheimer's disease pathogenesis by transporting amyloid beta to the blood-brain barrier, activating proinflammatory genes, including IL-6. ${ }^{25,38,39}$ A recent study reported that hippocampal RAGE expression was enhanced in a time-dependent manner after acute BCCAo. ${ }^{40}$ It is therefore expected that downregulation of Ang-II and RAGE could be beneficial in CCH. Our Western blot analysis showed a significant decrease in Ang-II and RAGE expressions in rats treated with E. F. mume following chronic BCCAo. Taken together, these data suggest that F. mume attenuates neuroinflammation by modulating Ang-II and RAGE signaling. In addition, oxidative stress is thought to play a major role in the pathogenesis of $\mathrm{CCH}^{4}{ }^{41,42}$ It has been reported that oxidative event-related by-products, such as reactive oxygen species and malondialdehyde, are produced in rats following BCCAo. ${ }^{43,44}$ Further studies are needed to examine the antioxidative effects of $F$. mume administration after BCCAo.
Central cholinergic dysfunction underlies many learning and memory disturbances in patients with $\mathrm{VaD} .{ }^{45}$ Increasing evidence has suggested that $\mathrm{CCH}$ may reduce $\mathrm{ChAT}$ expression, leading to decreased $\mathrm{ACh}$ synthesis and contributing to cognitive impairment. ${ }^{13,46,47}$ In the present study, we found that E. F. mume prevents the BCCAo-induced dysregulation of ChAT. These results suggest that $F$. mume might protect the cholinergic system against $\mathrm{CCH}$. Since ChAT expression could be increased by members of the neurotrophin family, ${ }^{48}$ further research is needed to investigate the upstream mechanisms resulting in increased ChAT expression following $F$. mume treatment.

Natural medicinal foods, also called nutraceuticals, which provide neuroprotection to people with dementia, have been the focus of many recent studies. ${ }^{49}$ For example, curcumin, a herb of the ginger family, lowered levels of proinflammatory cytokines and oxidized proteins in a transgenic Alzheimer's disease mouse model. ${ }^{50}$ Soybean administration protected mice from scopolamine-induced memory impairment and oxidative stress. Treatment of BCCAo rats with a water extract of Triticum aestivum L. effectively attenuated changes in myelin basic protein and GFAP. ${ }^{51}$ Since $F$. mume exerts similar effects, we suggest that $F$. mume also has potential as a neuroprotective nutraceutical against $\mathrm{VaD}$.

In conclusion, we demonstrated that E. F. mume alleviates the inflammatory responses and cholinergic dysfunction induced by chronic BCCAo. Along with our earlier reports, we, therefore, hypothesize that $F$. mume might ameliorate neurological dysfunction and cognitive impairment following $\mathrm{CCH}$ by preventing inflammation and improving the function of the cholinergic system.

\section{ACKNOWLEDGMENTS}

This work was supported by a grant (KIOM-2010-2) from the Inter-Institutional Collaboration Research Program under the Korea Research Council of Fundamental Science \& Technology. This study was also supported, in part, by grants (K12220, K13220, and K15310) from the KIOM.

\section{AUTHOR DISCLOSURE STATEMENT}

No competing financial interests exist.

\section{REFERENCES}

1. Cumming T, Brodtmann A: Dementia and stroke: The present and future epidemic. Int J Stroke 2010;5:453-454.

2. Versijpt J: Effectiveness and cost-effectiveness of the pharmacological treatment of Alzheimer's disease and vascular dementia. J Alzheimers Disease 2014;42:S19-S25.

3. Iadecola C: The pathobiology of vascular dementia. Neuron 2013;80:844-866.

4. Zhao Y, Gu JH, Dai CL, et al.: Chronic cerebral hypoperfusion causes decrease of O-GlcNAcylation, hyperphosphorylation of tau and behavioral deficits in mice. Front Aging Neurosci 2014;6:10.

5. Otori T, Katsumata T, Muramatsu H, Kashiwagi F, Katayama Y, Terashi A: Long-term measurement of cerebral blood flow and 
metabolism in a rat chronic hypoperfusion model. Clin Exp Pharmacol Physiol 2003;30:266-272.

6. Bang J, Jeon WK, Lee IS, Han JS, Kim BY: Biphasic functional regulation in hippocampus of rat with chronic cerebral hypoperfusion induced by permanent occlusion of bilateral common carotid artery. PLoS One 2013;8:e70093.

7. Lee KM, Bang JH, Han JS, et al.: Cardiotonic pill attenuates white matter and hippocampal damage via inhibiting microglial activation and downregulating ERK and p38 MAPK signaling in chronic cerebral hypoperfused rat. BMC Complement Altern Med 2013;13:334.

8. Gupta A, Watkins A, Thomas P, et al: Coagulation and inflammatory markers in Alzheimer's and vascular dementia. Int $J$ Clin Pract 2005;59:52-57.

9. Zuliani G, Ranzini M, Guerra G, et al.: Plasma cytokines profile in older subjects with late onset Alzheimer's disease or vascular dementia. J Psychiatr Res 2007;41:686-693.

10. Picciotto MR, Higley MJ, Mineur YS: Acetylcholine as a neuromodulator: Cholinergic signaling shapes nervous system function and behavior. Neuron 2012;76:116-129.

11. Schliebs R, Arendt T: The cholinergic system in aging and neuronal degeneration. Behav Brain Res 2011;221:555-563.

12. Sharma B, Singh N: Experimental hypertension induced vascular dementia: Pharmacological, biochemical and behavioral recuperation by angiotensin receptor blocker and acetylcholinesterase inhibitor. Pharmacol Biochem Behav 2012;102:101-108.

13. Choi BR, Kwon KJ, Park SH, et al:: Alternations of septalhippocampal system in the adult Wistar rat with spatial memory impairments induced by chronic cerebral hypoperfusion. Exp Neurobiol 2011;20:92-99.

14. Kwon KJ, Kim MK, Lee EJ, et al.: Effects of donepezil, an acetylcholinesterase inhibitor, on neurogenesis in a rat model of vascular dementia. J Neurol Sci 2014;347:66-77.

15. Chuda Y, Ono H, Ohnishi-Kameyama M, Matsumoto K, Nagata T, Kikuchi Y: Mumefural, citric acid derivative improving blood fluidity from fruit-juice concentrate of Japanese apricot (Prunus mume Sieb. et Zucc). J Agric Food Chem 1999;47:828-831.

16. Choi HJ, Kang OH, Park PS, et al: : Mume Fructus water extract inhibits pro-inflammatory mediators in lipopolysaccharidestimulated macrophages. J Med Food 2007;10:460-466.

17. Jin H, Lee B, Lim K, Debnath T, Shin H, Lim B: Antiinflammatory effects of Prunus mume mixture in colitis induced by dextran sodium sulfate. Korean J Med Crop Sci 2011;19: $16-23$.

18. Kim MS, Jeon WK, Lee KW, Park YH, Han JS: Ameliorating effects of ethanol extract of Fructus mume on scopolamineinduced memory impairment in mice. Evid Based Complement Alternat Med 2015;2015:102734.

19. Jeon WK, Ma J, Choi BR, et al.: Effects of Fructus mume extract on MAPK and NF- $\kappa$ B signaling and the resultant improvement in the cognitive deficits induced by chronic cerebral hypoperfusion. Evid Based Complement Alternat Med 2012;2012:450838.

20. Nagai T, Myoda T, Nagashima T: Antioxidative activities of water extract and ethanol extract from field horsetail (tsukushi) Equisetum arvense L. Food Chem 2005;91:389-394.

21. Lee KM, Bang J, Kim BY, et al.: Fructus mume alleviates chronic cerebral hypoperfusion-induced white matter and hippocampal damage via inhibition of inflammation and downregulation of TLR4 and p38 MAPK signaling. BMC Complement Altern Med 2015;15:125.
22. Cho KO, La HO, Cho YJ, Sung KW, Kim SY: Minocycline attenuates white matter damage in a rat model of chronic cerebral hypoperfusion. J Neurosci Res 2006;83:285-291.

23. Nussbaum RL, Ellis CE: Alzheimer's disease and Parkinson's disease. N Engl J Med 2003;348:1356-1364.

24. Zhou J, Ando H, Macova M, Dou J, Saavedra JM: Angiotensin II AT1 receptor blockade abolishes brain microvascular inflammation and heat shock protein responses in hypertensive rats. $J$ Cereb Blood Flow Metab 2005;25:878-886.

25. Deane R, Wu Z, Zlokovic BV: RAGE (yin) versus LRP (yang) balance regulates alzheimer amyloid $\beta$-peptide clearance through transport across the blood-brain barrier. Stroke 2004;35:26282631.

26. Sartor RB: Microbial influences in inflammatory bowel diseases. Gastroenterology 2008;134:577-594.

27. Lin MC, Hui CF, Chen JY, Wu JL: The antimicrobial peptide, shrimp anti-lipopolysaccharide factor (SALF), inhibits proinflammatory cytokine expressions through the MAPK and NF- $\kappa \mathrm{B}$ pathways in Trichomonas vaginalis adherent to HeLa cells. Peptides 2012;38:197-207.

28. Williams B, Granholm AC, Sambamurti K: Age-dependent loss of NGF signaling in the rat basal forebrain is due to disrupted MAPK activation. Neurosci Lett 2007;413:110-114.

29. Veerapur V, Palkar M, Srinivasa H, et al.: The effect of ethanol extract of Wrightia tinctoria bark on wound healing in rats. $J$ Nat Remed 2004;4:155-159.

30. Sun J, Liu BR, Hu WJ, Yu LX, Qian XP: In vitro anticancer activity of aqueous extracts and ethanol extracts of fifteen taditional Chinese medicines on human digestive tumor cell lines. Phytother Res 2007;21:1102-1104.

31. Fukuda T, Ito H, Mukainaka T, Tokuda H, Nishino H, Yoshida T: Anti-tumor promoting effect of glycosides from Prunus persica seeds. Biol Pharm Bull 2003;26:271-273.

32. Seo CS, Kim JH, Shin HK: Simultaneous determination of seven constituents in herbal prescription jaeumganghwa-tang using HPLC-PDA. Afr J Tradit Complement Altern Med 2013;10:113123.

33. Shen H, Cheng T, Qiao C, Su Z, Li C: Antitumor effect in vitro and immuno-response in vivo of fructus Mume. Zhongguo Zhong Yao Za Zhi 1995;20:365-368.

34. Schmidt-Kastner R, Aguirre-Chen C, Saul I, et al:: Astrocytes react to oligemia in the forebrain induced by chronic bilateral common carotid artery occlusion in rats. Brain Res 2005;1052:28-39.

35. Rubio-Perez JM, Morillas-Ruiz JM: A review: Inflammatory process in Alzheimer's disease, role of cytokines. Sci World J 2012;2012:756357.

36. Guo RB, Wang GF, Zhao AP, Gu J, Sun XL, Hu G: Paeoniflorin protects against ischemia-induced brain damages in rats via inhibiting MAPKs/NF- $\kappa \mathrm{B}$-mediated inflammatory responses. PLoS One 2012;7:e49701.

37. Ishikawa M, Sekizuka E, Yamaguchi N, et al.: Angiotensin II type 1 receptor signaling contributes to platelet-leukocyteendothelial cell interactions in the cerebral microvasculature. Am J Physiol 2007;292:H2306-H2315.

38. Takuma K, Fang F, Zhang W, et al.: RAGE-mediated signaling contributes to intraneuronal transport of amyloid-beta and neuronal dysfunction. Proc Natl Acad Sci USA 2009;106:20021-20026.

39. Bierhaus A, Schiekofer S, Schwaninger M, et al.: Diabetesassociated sustained activation of the transcription factor nuclear factor- $\kappa$ B. Diabetes 2001;50:2792-2808. 
40. Kamide T, Kitao Y, Takeichi T, et al:: RAGE mediates vascular injury and inflammation after global cerebral ischemia. Neurochem Int 2012;60:220-228.

41. Farkas E, Luiten PG, Bari F: Permanent, bilateral common carotid artery occlusion in the rat: A model for chronic cerebral hypoperfusion-related neurodegenerative diseases. Brain Res Rev 2007;54:162-180.

42. Cai ZY, Yan Y, Sun SQ, et al:: Minocycline attenuates cognitive impairment and restrains oxidative stress in the hippocampus of rats with chronic cerebral hypoperfusion. Neurosci Bull 2008;24: 305-313.

43. Aytac E, Seymen HO, Uzun H, Dikmen G, Altug T: Effects of iloprost on visual evoked potentials and brain tissue oxidative stress after bilateral common carotid artery occlusion. Prostaglandins Leukot Essent Fatty Acids 2006;74:373-378.

44. Ghoneim AI, Abdel-Naim AB, Khalifa AE, El-Denshary ES: Protective effects of curcumin against ischaemia/reperfusion insult in rat forebrain. Pharmacol Res 2002;46:273-279.

45. Wang J, Zhang HY, Tang XC: Cholinergic deficiency involved in vascular dementia: Possible mechanism and strategy of treatment. Acta Pharmacol Sin 2009;30:879-888.
46. Zhao RR, Xu F, Xu XC, et al.: Effects of $\alpha$-lipoic acid on spatial learning and memory, oxidative stress, and central cholinergic system in a rat model of vascular dementia. Neurosci Lett 2015;587:113-119.

47. Xi Y, Wang M, Zhang W, et al.: Neuronal damage, central cholinergic dysfunction and oxidative damage correlate with cognitive deficits in rats with chronic cerebral hypoperfusion. Neurobiol Learn Mem 2014;109:7-19.

48. Burgess A, Aubert I: Polysialic acid limits choline acetyltransferase activity induced by brain-derived neurotrophic factor. J Neurochem 2006;99:797-806.

49. Mecocci P, Tinarelli C, Schulz R, Polidori M: Nutraceuticals in cognitive impairment and Alzheimer's disease. Front Pharmacol 2014;5:147.

50. Lim GP, Chu T, Yang F, Beech W, Frautschy SA, Cole GM: The curry spice curcumin reduces oxidative damage and amyloid pathology in an Alzheimer transgenic mouse. $J$ Neurosci 2001;21:8370-8377.

51. Bansal N, Parle M: Soybean supplementation helps reverse ageand scopolamine-induced memory deficits in mice. J Med Food 2010;13:1293-1300. 